\section{Questión}

Periodismo / Comunicación ISSN 1669-6581

Ensayo en defensa de la ontología

Gabriel Miranda

Question/Cuestión, Nro.67, Vol.2, diciembre 2020

ISSN: 1669-6581

URL de la Revista: https://perio.unlp.edu.ar/ojs/index.php/question/

IICom - FPyCS - UNLP.

DOI: https//doi.org/10.24215/16696581e408

\title{
Ensayo en defensa de la ontología
}

\section{Ontology must be defended}

\section{Gabriel Miranda}

Universidade Federal do Rio Grande do Norte; École des hautes études en sciences sociales

Francia

g.m.b94@hotmail.com https://orcid.org/0000-0002-3331-2890

\section{Resumen}

El presente trabajo, de carácter exploratorio, propone un ejercicio filosófico sobre el proceso de construcción del conocimiento. En un momento histórico marcado por el relativismo y el subjetivismo, cuyas expresiones más radicales 
aparecen en movimientos como el "terraplanismo", este ensayo propone el rescate de una producción ontológica del conocimiento, o sea, centrado en lo real y no en las múltiples interpretaciones posibles que lo real puede adquirir. Con este fin, se recuperan las contribuciones de autores clásicos como Karel Kosík, José Chasin, Karl Marx y Friedrich Engels. Del mismo modo, el texto también destaca la necesidad de superar la separación entre la producción científica y la acción política, señalando la necesidad de una ciencia dedicada a un proyecto de transformación social en beneficio de aquellos y aquellas quienes, en la sociabilidad burguesa, son explotados y oprimidos.

\section{Palabras clave}

dialéctica; ontología; pseudoconcreción; marxismo; método.

\section{Abstract}

This paper proposes a philosophical exercise about the process of construction of scientific knowledge. In a historical moment marked by relativism and subjectivism, whose more radical expressions appear in movements such as "The Flat Earth", this essay proposes the rescue of a method scientific based on ontological perspective, that is, centered on the real and not on the multiple interpretations that the real can acquire. To this end, contributions from classical authors such as Karel Kosík, José Chasin, Karl Marx and Friedrich Engels are recovered. Likewise, the text also indicates the need to overcome the separation between scientific production and political action, pointing out the need for a science dedicated to a project of social transformation for the benefit of those who, in bourgeois sociability, are exploited and oppressed. 


\section{Keywords}

dialectics; ontology; pseudoconcrete; Marxism; method.

\section{Introducción}

Esta ponencia teórica y exploratoria constituye el campo de la Filosofía de la Ciencia y busca demarcar la perspectiva ontológica de la construcción del conocimiento. Por lo tanto, para contrarrestar el idealismo y el relativismo que se apoderan de las Ciencias Humanas, se argumenta que el conocimiento científico debería adoptar como base principal la primacía ontológica de lo real. Admitir tal presupuesto no significa creer que la realidad social tiene una esencia inmutable y depende del investigador social revelarla, como creen los que abogan por una ontología metafísica. Por el contrario, la realidad social es producto de la acción de hombres y mujeres y, como tal, asume un carácter histórico. En ese sentido no puede haber esencia inmutable.

Sin embargo, al defender la ontología sobre la gnosiología, nos referimos a una ontología sociohistórica, inspirada sobre todo en la forma marxista de proceder científicamente. Esto significa que, en primer lugar, se supone que la realidad tiene un carácter ontológico, es decir, existe independientemente de lo que el sujeto cree que es la realidad. En segundo lugar, la realidad no puede entenderse únicamente a través de los órganos sensoriales. Por esta razón, la capacidad de abstracción del investigador es necesaria para superar la apariencia fenoménica de la realidad.

A su vez, superar el nivel de la pseudoconcreción, que se presenta a través de los órganos sensoriales, significa identificar los procesos históricos que hicieron que el "objeto" en análisis asumiera la forma que presenta. Por lo tanto, podemos suponer que el criterio de cientificidad desde la perspectiva 
ontológica sociohistórica reside en la historia, específicamente en la precisión de demostrar paso a paso los procesos históricos que determinan el ser.

Finalmente, el texto señala la necesidad de un conocimiento que se dirige a un proyecto de transformación social en beneficio de los explotados y oprimidos en la sociabilidad burguesa. En el libro Pedagogía del oprimido, escrito por el intelectual brasileño Paulo Freire, se lee: «a los desharrapados del mundo y a quienes, descubriéndose en ellos, con ellos sufren, y con ellos luchan» (Freire 2005 , p. 9). Los tres verbos en la afirmación anterior proporcionan la síntesis del esfuerzo intelectual crítico: descubrir, sufrir, luchar.

Para descubrir, o sea, revelar la realidad que captamos a través de los sentidos, es necesario la ciencia. Al descubrir los engranajes de la realidad en los que estamos inmersos, sufrimos. Tal sufrimiento revela el carácter éticopolítico de la acción científica, porque al comprender el mundo, nos entendemos a nosotros mismos $y$, así, nos indignamos ante la injusticia y sufrimos. Pero no es un sufrimiento melancólico y encarcelador, sino un sufrimiento insubordinado e insurgente, que impulsa la lucha por construir un mundo nuevo. En este sentido, tenemos el trío dialéctico descubrir-sufrir-luchar que caracterizan las producciones científicas que están comprometidas no con el mantenimiento de la realidad social, sino con su transformación.

\section{Notas acerca de la construcción dialéctica del conocimiento}

En 1963, se publica por primera vez el libro Dialéctica de lo concreto, originado a partir de dos conferencias impartidas en el año de 1960 por el filósofo checo Karel Kosík. En este libro hay algunas nociones fundamentales sobre la construcción del conocimiento científico desde la perspectiva ontológica y es precisamente por esta razón que esta obra se evoca en este punto. 
Comencemos por referencia a la primera afirmación del texto: «la dialéctica trata de la "cosa misma". Pero la "cosa misma" no se manifiesta inmediatamente al hombre» (Kosík 1984, p. 9).

Primero, asumir el presupuesto que la dialéctica se ocupa de la cosa en sí significa que hay "una cosa en sí misma" para conocer. En segundo lugar, para la dialéctica, es importante conocer "la cosa misma", no las múltiples interpretaciones que la "cosa misma" tiene para los seres humanos. Por lo tanto, se hace una distinción desde esta perspectiva en relación con las perspectivas relativistas, actualmente hegemónicas en las Ciencias Sociales (Tonet, 2013). Es cierto que, como seres sociales, la forma en que interpretamos la realidad está determinada por los marcadores sociales que definen nuestra posición en la sociedad, como raza, genero, orientación sexual, clase etc. Sin embargo, esto no significa que nuestras interpretaciones múltiples y circunstanciales - aborden lo real, la "cosa misma" a la que se refiere Kosík. Por el contrario, son "representaciones de la cosa".

Para la perspectiva dialéctica de la construcción del conocimiento, es vital la diferencia entre la "cosa misma" y la "representación de la cosa". Si pensamos en la construcción del conocimiento desde el binomio sujeto-objeto, el polo fundamental de la producción dialéctica del conocimiento reside en el objeto, no en el sujeto, conforme se consolidó con la Ciencia Moderna (Tonet 2013). Por ejemplo, el daltónico ve el rosa como blanco, pero eso no hará que el blanco sea realmente color rosa. Es decir, es importante conocer la realidad tal como es, y no solamente los significados que el sujeto le atribuye. ¿Pero cómo emprender esa tarea de conocer la realidad tal como es? ¿Y cuál es el criterio de cientificidad que asegurará que uno conozca la "cosa misma" y ya no sea 
una representación de la realidad investigada? Estas son algunas de las preguntas que esta ponencia busca responder.

Lograr la "cosa misma" se trata de emprender una búsqueda de la esencia del fenómeno, es decir, una producción de conocimiento ontológico. Pero no se trata de una ontología metafísica, ahistórica, como en el modelo de construcción del conocimiento greco-medieval, donde se buscaba descubrir la esencia inmutable de las cosas, como hicieron los presocráticos, Platón, Aristóteles y los intelectuales de la Edad Media, como San Agustín y Santo Tomás de Aquino (Chasin 1988). Debido al carácter dialéctico e histórico de la realidad, la ontología es sociohistórica, no metafísica, como querían los griegos y los pensadores de la Edad Media. De lo contrario, estaríamos condenados a un determinismo conservador del orden establecido. Sin embargo, con la consolidación de la Ciencia Moderna, la perspectiva ontológica, centrada en la objetividad, se dejó de lado y la perspectiva gnosiológica, centrada en la subjetividad, se volvió hegemónica.

Como consecuencia de este proceso, se ha perdido la dimensión ontológica del hacer científico, lo que afirma que la realidad es importante en sí misma, independientemente de generar subjetividad. En este sentido, el conocimiento sin ontología, sin una esencia que concierne a la cosa misma y no a las interpretaciones de los sujetos, no puede ir más allá del universo de opiniones particulares. Para ilustrar el debate sobre la primacía ontológica de lo real, tomemos como objeto el cultivo de una planta. No es el científico quien atribuye que el agua es importante para la planta, es la planta en su existencia que informa que sin agua no puede desarrollarse. El papel del científico es descubrir las articulaciones objetivas entre el agua y la planta, no crearlas (Chasin, 1988). 
Finalmente, retomando la afirmación inicial de Kosík (1984) de que la "cosa en sí" no se manifiesta directamente al género humano, Karel Kosík (1984) señala el carácter limitado de las perspectivas gnosiológicas, que creen que agotan la realidad a través de los órganos sensoriales. Para la perspectiva marxista de la construcción del conocimiento, los datos empíricos a los que se accede a través de los órganos de los sentidos, es decir, los datos cualitativos obtenidos en entrevistas, los diarios de campo producidos a través de observaciones y las estadísticas producidas sobre fenómenos sociales, nos brindan acceso a una dimensión parcial de la realidad. Tal dimensión parcial de la realidad, que Karel Kosík (1984) llama pseudoconcreción o "apariencia", no es falsa, pero es parcial, porque no tiene en cuenta las determinaciones históricas involucradas en la constitución de lo real. Por lo tanto, se debe suponer que, además de la apariencia fenoménica, que se presenta directamente al género humano, existe una "esencia de la cosa" a la que no se puede acceder únicamente por medio de los órganos sensoriales, dado que a través de estos no avanzamos más allá del nivel de pseudoconcreción. Según Oliveira y Costa (2018, p. 220):

En nuestra vida cotidiana, todo el tiempo nos encontramos con varios objetos y situaciones sociales que nos aparecen en forma de fenómeno. ¿Pero qué es esto? El fenómeno (o el singular) es algo que nos viene a la vista de inmediato, pero sin lo que Kosík (2002) Ilamó determinaciones. El fenómeno pertenece al Real, pero no es lo mismo que el real. Es un fragmento que revela lo Real mientras lo oculta. El fenómeno, separado de lo Real, lleva en sí una singularidad y aislamiento que nos impide de comprender por qué él existe, para qué existe, al servicio de qué/quién él existe. Lo fenómeno es una sección 
del Real mediada por la ideología (en el sentido lukacsiano). Así, si aprehendemos los hechos de la vida sin prestar atención a sus determinaciones, que, para Marx, son históricas, tendemos a absorber estos fenómenos suave y "naturalmente", como si fueran el curso fatal de la vida y sin establecer relaciones entre los objetos sociales. (1)

Para entender la realidad tal como es, es decir, pasar de la pseudoconcreción al concreto, superar el nivel de apariencia fenoménica y alcanzar la esencia de la cosa, es necesario, en palabras de Kosík (1984), realizar un "détour". Esto significa que el científico social debe tratar de responder la siguiente pregunta: "¿Qué determinaciones fundamentales permiten que la realidad se presente de esta manera y no de otra?". Por lo tanto, el método dialéctico de construcción del conocimiento presupone el rescate de procesos - sean ellos políticos, sociales, económicos o culturales - involucrados en la determinación del fenómeno investigado. Por eso, en la ldeologia alemã (2007, p. 86), Marx y Engels afirman que "solo hay una ciencia, la ciencia de la historia"(2), pues para el materialismo histórico-dialéctico, analizar la realidad implica necesariamente rehacer la red de procesos históricos involucrados en la determinación del presente $\mathrm{y}$, además, tratar de comprender cómo están interconectados los fenómenos del presente, de modo que investigar cualquier fenómeno social presupone buscar relaciones que no están colocadas de inmediato. Como señala Karel Kosík (1984, p.12-13):

La dialéctica es el pensamiento crítico que quiere comprender la "cosa misma", y se pregunta sistemáticamente cómo es posible llegar a la comprensión de la realidad. Es, pues, lo opuesto a la sistematización 
doctrinaria o a la romantización de las representaciones comunes. El pensamiento que quiera conocer adecuadamente la realidad, y que no se contente con los esquemas abstractos de la realidad, ni con simples representaciones también abstractas de ella, debe destruir la aparente independencia del mundo de las relaciones inmediatas cotidianas. El pensamiento que destruye la pseudoconcreción para alcanzar lo concreto es, al mismo tiempo, un proceso en el curso del cual bajo el mundo de la apariencia se revela el mundo real; tras la apariencia externa del fenómeno se descubre la ley del fenómeno, la esencia. Pero lo que confiere a estos fenómenos el carácter de la pseudoconcreción no es de por sí su existencia, sino la independencia con que esta existencia se manifiesta. La destrucción de la pseudoconcreción, que el pensamiento dialéctico debe llevar a cabo, no niega por ello la existencia u objetividad de estos fenómenos, sino que destruye su pretendida independencia al demostrar que son causa mediata y, contrarrestando sus pretensiones de independencia, prueba su carácter derivado.

Por ejemplo, según datos del Atlas da Violência 2019, en Brasil, en el año de 2017 , el $75,5 \%$ de las 65.602 víctimas de homicidio eran negras mientras 55\% de la población brasileña era negra. (Cerqueira, 2019). Para analizar la representación excesiva de la población negra en las altas tasas de homicidio en Brasil, es necesario tener en cuenta el proceso histórico de formación del estado brasileño, marcado por más de trescientos años de esclavitud de negros y una abolición formal que no instituye ningún mecanismo para la inserción de la población negra en la sociedad de clases (Florestan, 2008). De 
tal manera, mirando al pasado, es posible ver por qué la población negra constituye los sectores más empobrecidos de la clase trabajadora brasileña.

Además, comprender los altos niveles de violencia también permea el análisis del modo de producción capitalista y sus particularidades en el contexto latinoamericano, teniendo en cuenta, incluso, los mecanismos de gestión penal de la miseria en un escenario de avance del neoliberalismo, la política prohibicionista de drogas y la militarización de la policía. Por lo tanto, la tarea elemental del materialismo histórico-dialéctico consiste en darse cuenta de que el fenómeno no termina consigo mismo y, por esta razón, es necesario comprender lo que está dialécticamente relacionado en su producción y reproducción.

Por eso, para la construcción dialéctica del conocimiento, la totalidad es uno de sus elementos centrales. Sin embargo, la noción del análisis de la realidad desde la totalidad ha sido mal interpretada por una parte considerable de las Ciencias Humanas, que insiste que los marxistas proponen construir verdades absolutas en su análisis. Por el contrario, debido al carácter dialéctico de la realidad, es decir, en proceso constante de transformación, el proceso de totalización nunca termina, de modo que los análisis propuestos siempre se refieren a una realidad pasada. Por lo tanto, tomar la totalidad como presupuesto significa el esfuerzo constante para comprender que los objetos investigados constituyen y están constituidos por un determinado contexto, y no están aislados en un vacío de tiempo y espacio. Como Konder (2008, p. 36) advierte:

Cualquier objeto que el hombre pueda percibir o crear es parte de un todo. En cada acción tomada, el ser humano se choca, inevitablemente, 
con problemas interconectados. Por esta razón, para encaminar una solución para los problemas, el ser humano necesita tener una cierta visión del conjunto de ellos: es desde esta mirada que nosotros podemos evaluar la dimensión de cada elemento del cuadro. Eso fue lo que subrayó Hegel cuando escribió: "La verdad es el todo". Si no vemos el todo, podemos atribuir un valor exagerado a una verdad limitada (convirtiéndola en una mentira), dañando nuestra comprensión de una verdad más general. (3)

Para Karel Kosík (1984) y para la producción dialéctica del conocimiento, es importante destruir la pseudoconcreción, no porque sea falso, sino porque es parcial. La crítica emprendida es que los datos empíricos representan el punto de partida de la construcción del conocimiento crítico, no su etapa final. A partir de los datos empíricos, es necesario ejercer la abstracción, buscando articular aquellas determinaciones que, aunque involucradas en la determinación de lo real, no son aparentes. Una vez que se identifican estas determinaciones, volvemos a lo empírico. Por lo tanto, en la investigación de inspiración marxista, lo empírico aparece como un punto de inicio y fin, pero más importante que el principio y el final es el camino, la búsqueda de las determinaciones fundamentales del objeto investigado, el llamado détour.

Es cierto que la búsqueda de las mediaciones y contradicciones del fenómeno investigado siempre será limitada, ya sea porque la realidad está en un proceso constante de transformación y nuestros análisis siempre están relacionados con algo que ya sucedió, o porque, como sujetos, tenemos limitaciones de tiempo, recursos y apropiación del marco teórico y conceptual necesario para el análisis. Por lo tanto, siguiendo el camino señalado por Pedro Demo (1995), 
parece apropiado adoptar la discutibilidade como criterio de cientificidad, es decir, hacer evidente el método empleado para lograr los resultados expuestos en alguna investigación, de modo que los interlocutores de la investigación puedan juzgar la razonabilidad de la producción. También hay objeciones de que el marxismo sería una perspectiva economista y determinista. Frente a esto, el siguiente extracto de Engels es quirúrgico para combatir las distorsiones mecanicistas impuestas al materialismo histórico-dialéctico, especialmente después de la Segunda Internacional:

Según la concepción materialista de la historia, el factor que en última instancia determina la historia es la producción y la reproducción de la vida real. Ni Marx ni yo hemos afirmado nunca más que esto. Si alguien lo tergiversa diciendo que el factor económico es el único determinante, convertirá aquella tesis en una frase vacua, abstracta, absurda. La situación económica es la base, pero los diversos factores de la superestructura [...] ejercen también su influencia sobre el curso de las luchas históricas y determinan, predominantemente en muchos casos, su forma. (Marx y Engels 1974, p. 1)

De este modo, en Marx, el sujeto no es pasivo. Como bien se afirma en El Dieciocho Brumario de Luis Bonaparte: «los hombres hacen su propia historia, pero no la hacen a su libre arbitrio, bajo circunstancias elegidas por ellos mismos, sino bajo aquellas circunstancias con que se encuentran directamente, que existen y les han sido legadas por el pasado» (4) (Marx, 2011, p. 25). Por lo tanto, es un sujeto activo, pero su acción está limitada por las condiciones materiales de existencia. Esta es la razón por la cual la categoría de trabajo es central para Marx, porque a través del trabajo la especie humana transforma la 
naturaleza y a sí misma. El materialismo mecanicista y cualquier otra perspectiva determinista interpretan al sujeto como un ser pasivo $\mathrm{y}$, por lo tanto, propone análisis conservadores. Al contrario, en el marxismo, el sujeto está si sujeto a estructuras sociales, pero también es sujeto de sí mismo y puede actuar en el proceso de reproducción o transformación de la sociedad.

\section{Reflexiones finales}

Es ampliamente conocido por todos aquellos involucrados en los debates sobre la fundación de la Sociología que esta área de conocimiento se ha definido como una ciencia sin madre, pero hija de tres padres: el francés Émile Durkheim y los alemanes Max Weber y Karl Marx. A diferencia de Émile Durkheim (2001) y Max Weber (2001), Karl Marx no escribió ningún trabajo en que buscara definir el objeto y el método de la Sociología. La razón de esta opción es que el compromiso de este autor no fue demarcar la Sociología como área científica, sino comprender la génesis, el desarrollo y las posibilidades de superar la sociedad capitalista. Por lo tanto, la dimensión política siempre ha estado presente en el trabajo de Marx. Si, por un lado, Durkheim y Weber se preocupaban por analizar las transformaciones que marcaron el siglo XIX, Karl Marx quería tanto analizar las transformaciones del siglo XIX cuanto entender la sociabilidad burguesa como superarla. En este sentido, no es posible separar al teórico del hombre de acción y la forma más adecuada de conocer su método es comprender, en la lectura de sus obras, la forma en que este autor procedió.

Por lo tanto, Marx es el autor que funda una ciencia que se ocupa no solo de comprender la sociedad sino de transformarla. Es con él que nace la llamada "teoría crítica", como Max Horkheimer, un exponente de la Escuela de 
Frankfurt, propuso en el texto "Teoría tradicional y teoría crítica", publicado originalmente en 1937. Para Horkheimer (1983), hay al menos dos tipos de producción de conocimiento científico, las cuales llamó de teoría tradicional y, en el lado opuesto, la llamada teoría crítica, que, en contraste con los axiomas positivistas, se distingue de la teoría tradicional y afirma que la realidad es el producto de la acción humana y, por lo tanto, puede ser transformada.

En este sentido, una tarea esencial para todos los investigadores sociales que quieran ser críticos es entender la realidad social como un proceso en curso, o sea, entenderla como resultado de procesos históricos y, aún más, construir un conocimiento dirigido a un proyecto de transformación social dirigido a los explotados y oprimidos. La criticidad de las Ciencias Sociales radica precisamente en volver a conectar las dimensiones teóricas y prácticas, separadas por el ideal de la ciencia moderna y, por lo tanto, producir una ciencia que no naturalice la realidad y, además, permita descubrir las relaciones de explotación y opresión y señalar el camino de su superación.

\section{Notas}

(1) Traducción del autor. Fragmento original: «Na nossa vida cotidiana, o tempo todo nos deparamos com vários objetos e situações sociais que nos aparecem na forma de fenômeno. Mas, o que é isso? O fenômeno (ou o singular) é algo que nos chega aos olhos de forma imediata, mas desprovida do que Kosík (2002) chamou de determinações. O fenômeno pertence ao Real, mas não é a mesma coisa que ele. É um fragmento, que revela o Real ao mesmo tempo em que o oculta. O fenômeno, descolado do Real, carrega em si toda uma singularidade e um isolamento que nos impede de compreender porque ele existe, para que ele existe, a serviço de que/quem ele existe. Ele é um recorte 
do Real mediatizado pela ideologia (no sentido lukacsiano). Assim, se apreendemos os fatos da vida sem atentar para as suas determinações, que, para Marx, são históricas, tendemos a absorver esses fenômenos suave e "naturalmente", como se fossem o curso fatal da vida e sem estabelecer relações entre objetos sociais. "

(2) Traducción del autor. Fragmento original: «Só há uma ciência, a ciência da história»

(3) Traducción del autor. Fragmento original: «qualquer objeto que o homem possa perceber ou criar é parte de um todo. Em cada ação empreendida, o ser humano se defronta, inevitavelmente, com problemas interligados. Por isso, para encaminhar uma solução para os problemas, o ser humano precisa ter uma certa visão de conjunto deles: é a partir da visão do conjunto que a gente pode avaliar a dimensão de cada elemento do quadro. Foi o que Hegel sublinhou quando escreveu: 'A verdade é o todo.'. Se não enxergamos o todo, podemos atribuir um valor exagerado a uma verdade limitada (transformando-a em mentira), prejudicando a nossa compreensão de uma verdade mais geral. " (4) Traducción del autor. Fragmento original: "Os homens fazem a sua história. Contudo não a fazem de livre e espontânea vontade, mas sim de acordo com as circunstâncias que Ihes são legadas e transmitidas pelo passado»

\section{Referencias bibliográficas}

Cerqueira, D. et al. (2019). Atlas da Violência 2019. Instituto de Pesquisa Econômica Aplicada \& Fórum Brasileiro de Segurança Pública. Rio de Janeiro, Brasil. Recuperado de http://www.ipea.gov.br/portal/images/stories/PDFs/relatorio institucional/ 190605 atlas da violencia 2019.pdf 
Chasin, J. (1988). Superação do liberalismo. Maceió. Disponible en: https://www.marxists.org/portugues/chasin/1988/02/liberalismo.pdf

Demo, P. (1995). Metodologia científica em Ciências Sociais (3ª ed.). Brasil: Atlas.

Durkheim, E. (2001). As regras do método sociológico. (P. Nassetti, Trad.). Brasil: Martin Claret.

Fernandes, F. (2008). A integração do negro na sociedade de classes ( $2^{\circ}$ vol.). São Paulo: Globo.

Freire, P. (2005). Pedagogía del oprimido. (J. Mellado, Trad., 2ª ed.). México: Siglo XXI Editores S.A. Disponible en: https://fhcv.files.wordpress.com/2014/01/freire-pedagogia-deloprimido.pdf

Horkheimer, M. (1983). Teoria Tradicional e Teoria Crítica. In: W. Benjamin, M. Horkheimer, T. Adorno y J. Habermas. Textos escolhidos. (Col. Os Pensadores, v. XLVIII). (pp. 117-161). Brasil: Abril Cultural.

Konder, L. (2008). O que é dialética. São Paulo: Brasiliense.

Kosík, K. (1984). Dialéctica de lo concreto. (A. S. Vázquez, Trad., 10ªed.). México: Grijalbo. Disponible en: https://marxismocritico.files.wordpress.com/2012/05/dialecticadeloconcre to.pdf

Marx, K.; Engels, F. (1974). Obras Escogidas, en tres tomos (t. III), Moscú: Editorial Progreso. Disponible en: https://www.marxists.org/espanol/me/cartas/e21-9-90.htm

Marx, K. \& Engels, F. (2007). A ideologia alemã. São Paulo: Boitempo.

Marx, K. (2011). O 18 de Brumário de Luís Bonaparte. São Paulo: Boitempo. 
Oliveira, I. F.; Costa, A. L. F. (2018). O lugar do indivíduo na história: possíveis articulações entre Psicologia e Marxismo. In: I. F. Oliveira, I. L. Paiva, A. L. F. Costa et. al (Orgs.). Marx hoje: pesquisa e transformação social (pp. 213-228). São Paulo: Editora Expressão Popular.

Tonet, I. (2013). Método científico - uma abordagem ontológica. São Paulo: Instituto Lukács.Weber, M. (2001). Metodologia das Ciências Sociais. Parte I. (A. Wernet, Trad., 4르. ed). São Paulo: Cortez. 